\title{
LA KAHINA, LA REINE PALIMSESTE
}

\author{
Danielle Pister \\ Université de Lorraine (site de Metz)
}

\begin{abstract}
Résumé
La Kahina reste le personnage le plus emblématique de la résistance berbère à la conquête arabe du Maghreb, au viI ${ }^{\mathrm{e}}$ siècle. Paradoxalement, l'ignorance où nous sommes de sa véritable vie, faute de sources fiables, lui a conféré une aura qui lui a permis de continuer à incarner, jusqu'à nos jours, la longue et difficile histoire du Maghreb pour les différentes communautés qui ont vécu sur ce territoire et dont elle reflète la mémoire.
\end{abstract}

Mots clés : Algérie, Arabe, Aurès, Christianisme, Colonisation, Conquête arabe, Femme, Indépendance, Islam, Juif, Mémoire, Maghreb, Mort, Résistance, Tunisie.

\section{Resumen}

La Kahina sigue siendo el personaje más emblemático de la resistencia berberisca a la conquista árabe del Magreb, en el siglo vir. Paradójicamente, la ignorancia en la que nos encontramos de su verdadera vida, por falta de fuentes fiables, le confirió un aura que le permitió seguir encarnado, hasta nuestros días, la larga y difícil historia del Magreb para las distintas comunidades que vivieron en este territorio y cuya memoria refleja.

Palabras claves: Argelia, árabe, Aurès, cristianismo, colonización, conquista árabe, mujer, Independencia, Islam, judío, memoria (informe), el Magreb, muerte, resistencia, Túnez

La fortune éditoriale de la Kahina est inversement proportionnelle à ce que l'on sait de fiable sur le personnage. Quelques lignes suffisent aux historiens contemporains pour en rendre compte. ${ }^{1}$ En revanche, la fascination exercée par son destin reste encore forte aujourd'hui comme en témoignent les nombreux récits générés par une mémoire plurielle.

1 Dans l'Histoire de l'Afrique du Nord de Charles-André Julien, l'équipée de la Kahina occupe deux pages (p. 20-22) du second volume de la réédition parue à Paris, chez Payot en 1975. Bernard Lugan résume son combat en un paragraphe, à la page 70 de son Histoire des Berbères. Un combat identitaire plurimillénaire, Bernard Lugan Éditeur, 2012. Gilbert Meynier lui consacre un paragraphe et demi, p. 196-197, dans L'Algérie des origines. De la prébistoire à l'avènement de l'islam, La Découverte/Poche, 2010. 
Transmis d'abord oralement, avant d'être fixés par écrit, ils ont propagé une légende qui a transformé cette reine berbère en un véritable mythe.

$\mathrm{Au}$ sens étymologique, l'adjectif verbal latin legenda s'applique à un texte hagiographique "devant être lu ». La version écrite d'un récit, concernant des faits présentés comme véridiques, limite les interventions sur le corpus de celui qui en prend connaissance ou de celui qui se charge de le diffuser à un public assemblé à cet effet. La Légende dorée, recueil de vies de saints daté du XIII ${ }^{\mathrm{e}}$ siècle, qu'on lisait lors de la fête de l'un d'entre eux dans les monastères, pendant les repas, ou dans les églises pour édifier les fidèles, en donne un exemple. Au fil du temps, et en l'absence de témoignages irréfutables, auteurs et traducteurs surenchérirent sur les narrations précédentes afin de s'adapter à des publics nouveaux et pour mettre l'accent sur un aspect particulier négligé par les prédécesseurs. La vérité, en admettant qu'on la connût, s'embellit grâce à l'imagination féconde du conteur ou du scripteur. C'est ainsi que l'histoire de la Kahina, reine berbère ayant vécu à la fin du vi ${ }^{\mathrm{e}}$ siècle de notre ère, peut-être jusqu'au tout début du suivant, a gagné en ampleur au fil des siècles, au gré des communautés qui se sont emparées de sa mémoire. En s'éloignant du contexte originel, sa vie devint épopée et le personnage prit la dimension d'un mythe fondateur. Les actes qu'on lui prête furent à ce point magnifiés qu'ils devinrent exemplaires pour son peuple dont ils forgèrent l'âme et le destin. Le passé glorieux éclaira leur présent, voire l'avenir auquel ils aspiraient.

La geste de la Kahina, colportée d'abord oralement, a traversé les siècles grâce aux érudits qui ont transcrit ses exploits, avant que les romanciers ne se laissent séduire par cette reine berbère et ne la transforment en une « reine-palimpseste ». ${ }^{2} \mathrm{Si}$ les chroniques concernant la Kahina se recoupent sur l'essentiel, elles diffèrent sur certains points, parfois importants. Il n'existe pas de témoignages contemporains de son existence, ni de vestiges matériels attestant sa présence à une date et en un lieu précis. ${ }^{3}$ Aucune effigie d'elle ne nous est parvenue, ce qui n'étonne

2 Du grec ancien $\pi \alpha \lambda i \mu \psi \eta \sigma \tau o \varsigma$ / palímpsêstos, « gratté de nouveau »), ce terme désigne un manuscrit constitué d'un parchemin déjà utilisé, dont on a fait disparaitre les inscriptions visibles pour pouvoir y écrire un nouveau texte. Cette pratique fut surtout courante entre les $\mathrm{VII}^{\mathrm{e}}$ et $\mathrm{XII}^{\mathrm{e}}$ siècles, chez les copistes. La cherté des parchemins explique la réutilisation d'anciens manuscrits.

3 Même si la ville de Bir El Atar - à 87 km de Tébessa, dans la région des Aurès en Algérie -, 
pas, s'agissant d'une civilisation nomade qui ne frappe pas de monnaie et communique, pour l'essentiel, oralement.

Les historiens antiques se sont désintéressés de l'Ifriqiya, ${ }^{4}$ après la fin des guerres puniques, en I46 av. J.-C. De la fin du vII ${ }^{\mathrm{e}}$ siècle jusqu'au milieu du $\mathrm{IX}^{\mathrm{e}}$ siècle, aucun texte ne rend compte de la conquête du Maghreb par les Arabes, ni de celle de l'Espagne, de la Sicile ou de l'Égypte. Il faut attendre la fin du XII ${ }^{\mathrm{e}}$ siècle pour que les chroniqueurs espagnols, portugais et arabes, s'intéressent aux dynasties arabes. Le manque de témoignages contemporains sur la Kahina a suscité des doutes sur son historicité, notamment chez l'érudit français Charles Le Beau (I7oII778)..$^{5}$ D'autres historiens virent, au contraire, dans la permanence de sa mémoire la preuve de son existence et du rôle qu'elle a joué dans les dernières années de la conquête arabe de l'Ifriqiya. ${ }^{6}$ Mais comment apprécier la fiabilité des témoignages parus plus d'un siècle et demi après la date présumée de sa mort et, pour la source essentielle, le Kitab al-Ibar (Le livre des exemples) d'Ibn Khaldoun, presque sept siècles plus tard ?7

Les chroniqueurs des conquêtes musulmanes des $\mathrm{VII}^{\mathrm{e}}$ et $\mathrm{VIII}^{\mathrm{e}}$ siècles, qui rapportent la résistance de la Kahina à cette avancée, enrichissent les textes antérieurs en rajoutant des détails non vérifiables. Pour les Berbères d'Afrique du Nord — qui utilisent plus volontiers, pour se désigner, le vocable d'Imazighen, « hommes libres » ou « nobles »-, ce personnage incarne la défense de leur identité, de leur langue, de leur culture. En témoignent encore aujourd'hui les échanges sur les réseaux sociaux qui

affirme abriter le célèbre Bir E1 Kahina (puits de la Kahina) où la reine berbère aurait été jetée par les Arabes après qu'ils l'eurent tuée, bien d'autres lieux portent ce nom. C'est dans la ville antique de Baghaï (wilaya de Kenchela), classée monument du patrimoine national, que se trouverait le château où aurait vécu la Kahina. A ucune fouille archéologique, à ce jour, n’a pu prouver son existence.

4 Province proconsulaire romaine, correspondant à peu près à l'actuelle Tunisie, plus une partie de l'Est de l'Algérie et de la Libye actuelle.

5 Auteur de l'Histoire du Bas-Empire en commençant à Constantin le Grand dont il rédige 21 des 27 volumes, publiés entre $1757-1811$.

6 Déformation de l'appellation latine Africa désignant la partie orientale du Maghreb médiéval, également orthographié Ifriqiyya (en berbère $<\left[\mathrm{HO}<Z S_{0}\right.$, en arabe إفريقية).

7 Auteurs et textes les plus souvent cités : Ibn 'Abd al-Ḥakam (803-871), Conquête de l'Égypte, de l'Afrique du Nord et de l'Espagne (vers 860) ; Al-Mālikī, Abū Bakr 'Abd lāh ibn Muhammad, Riyāạ al-Nufüs (Livre du jardin des âmes) vers 1072 ; Ibn Khaldoun (Tunis, 1332 - Le Caire, 1406), Histoire des Berbères et des dynasties musulmanes de l'Afrique septentrionale, traduite par le baron de Slane, Alger, imprimerie du Gouvernement, 1852. 
confrontent, parfois avec une grande vivacité, les opinions des internautes algériens, tunisiens, marocains ou français sur la Kahina. De nombreux restaurants, et autres boutiques, arborent son nom sur leurs enseignes dans tous les lieux où la diaspora berbère a fait souche. Mais cette ferveur n'est pas moindre dans les communautés juives, ayant vécu en Afrique du Nord jusque dans les années i96o. Ils considèrent également la Kahina comme une ancêtre illustre. Dans les deux cas, son image est marquée par une forte affectivité, liée à une mémoire identitaire et familiale. La référence, quoique plus faible, existe aussi chez les Français, installés en Algérie pendant I32 ans, car elle a servi à justifier, chez certains d'entre eux, leur présence sur cette terre.

C'est cette figure protéiforme, passée de l'histoire à la légende et devenue un mythe, sans jamais perdre totalement sa dimension politique, que nous essaierons d'éclairer. Il ne sera pas question pour autant de faire une enquête historique ${ }^{8}$ ni de nous livrer à une quête des textes aussi poussée que celle menée par Noureddine Sabri. ${ }^{9}$ Plus modestement, nous essaierons de dégager quelques étapes dans l'utilisation littéraire de ce personnage jusqu'à nos jours.

\section{LES DONNÉES HISTORIQUES}

L'histoire étant généralement écrite par les vainqueurs, on peut s'interroger sur les éléments qu'ils retiennent pour peindre leurs adversaires. Le récit est souvent peu informatif et oscille souvent entre apologie et philippique. Ibn-Khaldoun insiste sur les grandes qualités des Berbères qui « ont toujours été un peuple puissant, redoutable, brave et nombreux, comme tant d'autres dans ce monde, tels que les Arabes, les Persans, les Grecs et les Romains. » ${ }^{10} \mathrm{Il}$ ajoute : "On a vu chez les Berbères des choses tellement hors du commun, des faits tellement admirables, qu'il est impossible de méconnaître le grand soin que Dieu a eu de cette nation, l'extrême bonté qu'il lui a toujours témoignée, la combinaison de vertus

8 Nous nous réfèrerons à Gabriel Camps, qui a consacré sa vie à l'étude des Berbères, et à Yves Modéran, spécialiste du haut Moyen Âge en Afrique du Nord.

9 Sabri (Noureddine), La Kahéna. Un mythe à l’image du Maghreb, Paris, L'Harmattan, «Critiques littéraires ", 2011, $271 \mathrm{p}$.

10 Ibn Khaldoun, op. cit., p. 199. 
dont il l'a dotée, les nombreux genres de perfections auxquels il l'a fait atteindre et toutes les diverses qualités propres à l'espèce humaine qu'il lui a permis de réunir et de s'approprier. ${ }^{11}$ La vie de la Kahina illustre de façon exemplaire ces aptitudes hors norme. En fait, l'histoire de cette dernière n'est jamais traitée pour elle-même mais par rapport à la conquête arabe qu'il s'agit de magnifier comme l'« œuvre du Tout-Puissant » : on ne peut mépriser un peuple que Dieu a lui-même élu. D’ailleurs, la victoire des Arabes sera d'autant plus éclatante qu'elle est obtenue sur un adversaire redoutable.

Sans discuter la véracité des faits rapportés par les chroniques, on peut remarquer combien la personnalité et le destin prêtés à la reine berbère se confondent avec la longue histoire des peuples qui ont occupé auparavant l'Afrique du Nord. La mort au combat de ce personnage ne scelle pas seulement le destin d'une femme et de sa tribu, elle symbolise la disparition - même si, en réalité, elle ne fut accomplie que sur le long terme- de ce qui subsistait encore des éléments civilisationnels du pourtour méditerranéen dont ce territoire fut le creuset depuis la plus haute Antiquité. Rappelons que les Berbères sont les descendants des premiers occupants du territoire (vers 8500 av. J.-C.), dont on a retrouvé les traces dans la région de Capsa, la Gabsa de la Tunisie actuelle. Les Phéniciens fondent Carthage en 8I4 av. J.-c. L'activité commerciale de la cité inquiète les Romains qui finiront par la détruire pour s'établir progressivement sur ce territoire, jusqu'à ce les Vandales venus du Nord de l'Europe vers 430, soient à leur tour chassés par les Byzantins (Empire romain d'Orient), en 533. C'est au siècle suivant, peu après la mort du Prophète Mahomet à Médine en 632, que les incursions arabes vont commencer sur un territoire sétendant de la Lybie, à la Tunisie, et à l'Est $\mathrm{du}$ Constantinois actuels.

Cette histoire mouvementée constitue moins une succession de ruptures qu'une accumulation d'apports culturels : les Carthaginois sont largement hellénisés ; la colonisation romaine, très inégale, est menée par des Africains romanisés (certains devinrent des empereurs romains comme Septime Sévère, né à Septis Magna dans l'actuelle Libye); la romanité subsiste à travers les Vandales et les Byzantins se disent romains

11 Ibid., p. 202-203. 
(Roums). Le christianisme, qui s'installe entre la fin du premier et le début du second siècle, va trouver sur cette terre des émules qui joueront un rôle décisif dans l'histoire de l'Église. La personnalité de Saint Augustin d'Hippone, un Numide, donc un Berbère (354-430), résume l'importance de l'Afrique du Nord dans l'histoire des premiers siècles de l'Église à laquelle elle a donné, entre les deuxième et quatrième siècles, trois papes. Les Berbères des cités sont christianisés et romanisés. Les nomades de l'Ouest restent imprégnés par leurs pratiques ancestrales païennes. Ceux de l'Est, adoptent tous les schismes. Mais l'Église africaine, affaiblie par le donatisme qui a suscité une répression des Vandales, adeptes de l'arianisme, contre ce schisme, n'est plus en état d'empêcher l'implantation d'une nouvelle religion. Les Juifs, arrivés à des époques différentes, peut-être avec les Phéniciens, ou plus tard après la destruction du temple de Jérusalem (le premier au VI ${ }^{\mathrm{e}}$ siècle av. J.-C., le second en 70 sous Titus), se sont intégrés à des tribus berbères, à moins que certains de ces derniers ne se soient judaïsés. Enfin, il n'est pas exclu que les troupes des envahisseurs arabes soient accompagnées d'artisans juifs dont ils avaient notamment besoin pour la fabrication de leurs armes.

On peut comprendre que l'on puisse hésiter sur l'appartenance de la Kahina à tel groupe ethnique et à telle religion. L'onomastique offre un premier éclairage. Désignée par tous les chroniqueurs arabes par le surnom d'al-Kāhina, transcrit Kahina ou Kahena, ${ }^{12}$ on a rapproché la racine sémitique KHN de Kahin, le devin. Elle désignerait ici une prophétesse, voire une sorcière. Ce pouvoir, souvent attribué aux femmes dans des cultures païennes, était mis à contribution par les Arabes : ils se faisaient accompagner sur le champ de bataille par des devineresses, chargées de prédire l'issue des combats ou d'influer sur leur issue. Selon En-Noweïri, ${ }^{13}$ la Kahina " prédisait l'avenir et tout ce qu'elle annonça ne manqua pas d'arriver. " ${ }^{14}$ C'est ainsi, qu'avant la dernière bataille où elle sera tuée, et sur les « conseils dictés par les connaissances surnaturelles

12 Sauf s'il s'agit de respecter la graphie d'une citation, on gardera la graphie « Kahina », plus universelle.

13 Ibn 'Abd al-Hakam, dit En-Noweïri, mort vers 1331-33, auteur d'une encyclopédie dont la $5^{\mathrm{e}}$ partie est consacrée à l'histoire. Le baron de Slane en extrait la partie traitant de la guerre contre la Kahina qu'il ajoute, en appendice, à sa traduction de l'Histoire des Berbères d'Ibn Khaldoun.

14 Ibn Khaldoun, op. cit., Appendice, § XI, p. 340 (fragment du chroniqueur En-Noweïri). 
que ses démons familiers lui avaient enseignées ", elle annonce à ses fils sa mort prochaine et leur demande se rendre à l'ennemi avant le combat. ${ }^{15}$

Ibn Khaldoun précise l'identité et la généalogie de celle qu'il distingue parmi les «chefs les plus puissants » des Berbères, «la Kahena, reine du Mont-Auras, et dont le vrai nom était Dihya, fille de Tabeta, fils de Tifan. Sa famille faisait partie des Djeraoua, tribu qui fournissait des rois et des chefs à tous les Berbères descendus d'el-Ater. ${ }^{16}$ Comme il affirme que ces derniers pratiquaient le judaïsme, ${ }^{17}$ on en a déduit que la Kahena était juive. D'où l'identification de son surnom, réduit à ses seules consonnes KHN, à celui de Cohen. De l'hébreu כהן, titre conféré à Aaron, frère de Moïse de la tribu de Lévi, et à sa descendance masculine, signifiant " dévoués » au service du Temple de Jérusalem, notamment pour les sacrifices. Depuis la destruction du Temple, le nom a continué à se transmettre de père en fils. Pour Norbert Slousch, ${ }^{18}$ elle est la dernière représentante des chefs d'origine juive qui descendraient probablement « d'une famille de grands prêtres aaronides de Jérusalem ». Dans ce cas, Kahena désignerait une prêtresse, mais cette fonction est étrangère à toute pratique hébraïque. Dès I963, l'historien israélien H. Z. Hirschberg, en retraduisant le texte d'Ibn Khaldoun et en reprenant de manière rigoureuse l'ensemble du dossier, remit en cause cette interprétation, et de manière plus générale, l'existence même de grandes tribus berbères juives à la fin de l'Antiquité. On a rapproché aussi ce surnom du grec ancien Kópıvva / Kórinna, Corinne en français, « être pur ». En Afrique du Nord, toutes les prêtresses subissaient un rituel de purification, tradition sans doute d'origine animiste. Le Professeur tunisien M'hamed Hassine Fantar avance une autre hypothèse : «elle était kohenet au sens punique du terme... reine-prêtresse... de quelque divinité (païenne et guerrière) dont l'historiographie n'a pas retenu le nom ${ }^{19}$

15 Ibid., p. $214-215$ et p. 341.

16 Ibn Khaldoun, op. cit., p. 213.

17 Ibid., p. 208.

18 Slousch (Norbert), «La race d'El Cahena », in La Revue indigène, n 44, déc. 1909, p. 580-581.

19 On ne peut, pour plus de précisions, que renvoyer à l'étude d'Yves Modéran, « Kahena », in Encyclopédie berbère, 27 | Kairouan - Kifan Bel-Ghomari [En ligne], mis en ligne le 01 juin 2011, consulté le 25 avril 2018. uRL : http://journals.openedition.org/encyclopedieberbere/1306 
Par ailleurs, Dihya ( $\left.₫ \ll \Phi S_{\circ}\right)$, Diya, Dahya, rapproché de l'expression tamazighe (berbère) «Dyhia Tadmut " signifie « belle gazelle », les berbères étant souvent désignés par un sobriquet. On y a vu la déformation de Damiya ou Damya (devineresse) qui, peut-être n'est qu'un diminutif du nom latin Damiana (du grec Damía « Damia », déesse grecque de la fertilité, assimilée à Demeter). Tabeta a été corrigé en Matiya, Tifan en Théophane, ${ }^{20}$ ce qui peut renvoyer aussi bien au judaïsme qu'au christianisme. Or El-Maliki rapporte que lors de sa fuite ultime, « elle avait avec elle une énorme idole de bois qu'elle adorait ; on la portait devant elle sur un chameau ». Certains y ont vu une idole paienne, celle de Gurzil, une divinité amazighe représentée par un taureau. Si le culte du Taureau, symbole de virilité et de puissance, est connu en Afrique du Nord dans l'Antiquité, aucun élément historique ne prouve que Dihya en fut une prêtresse. D'autres parlent d'une statue du Christ, de la Vierge ou du saint patron de la reine. Ce qui amène Mohamed Talbi à y voir la preuve de son appartenance au christianisme. ${ }^{21}$ Déjà, Émile Masqueray (I843-I894), ${ }^{22}$ agrégé d'histoire, anthropologue, ethnologue, linguiste pratiquant l'arabe et plusieurs langues berbères, mettait en doute la judaïté de la Kahina car, pour lui, les Berbères descendraient des colons romains, dégradés et déchus. Pour lui, leur reine ne pouvait quêtre chrétienne.

Gabriel Camps et Yves Modéran s'accordent pour privilégier l'hypothèse d'une Kahina chrétienne, tout en reconnaissant l'absence de preuves irréfutables. ${ }^{23}$ Ils reprennent l'affirmation de Mohamed Talbi, selon laquelle l'Aurès était à cette époque christianisé, comme le prouvent de nombreux vestiges chrétiens. ${ }^{24} \mathrm{Au}$-delà, d'une vérité difficile à démontrer, cette multiplicité d'hypothèse, parfois exclusives les unes des autres, fait du personnage la résultante de toute l'histoire si complexe de l'Ifriqiya

20 Talbi (Mohamed), «Un nouveau fragment de l'histoire de l'Occident musulman l'épopée d'Al-Kahina ", in Les Cabiers de Tunisie, t. xIx, 1er et $2^{\mathrm{e}}$ trim., 1971, p. 19-52.

$21 \mathrm{Ibid}$.

22 Masqueray (Émile), Formation des cités chez les populations sédentaires de l'Algérie (Kabyles du Djurdjura, Chaiuïa de l'Aourâs, Beni Mezâb), Paris, E. Leroux, 1886 ; Éd. Fanny Colonna, Aix-enProvence, Edisud, 1983.

23 Camps (Gabriel), L'Afrique du nord au féminin, Librairie Académique Perrin, 1992, p. 124-139.

24 Modéran (Yves), « Kahena », in Encyclopédie berbère, 27 | Kairouan - Kifan Bel-Ghomari [En ligne], mis en ligne le 01 juin 2011, consulté le 25 avril 2018. URL : http://journals.openedition. org/encyclopedieberbere/1306 
jusqu'à la conquête arabe. Berbère, la Kahina appartient à la population qui la première a occupé le territoire ; l'ascendance chrétienne la relie aux Byzantins qui se voulaient héritiers des Romains, eux-mêmes vainqueurs des Phéniciens. Des trois fils qu'elle aurait eus, Ifran est supposé fils d'un Berbère, Yasdigan celui d'un Romain ("Grec »), et Khaled, le prisonnier arabe qu'elle a adopté. Cette fratrie établit ainsi un lien légitime, autant berbère qu'arabe, entre les trois. Cela explique l'ordre donnée par la Kahina pour qu'ils se rendent à Hassan, le chef Arabe : elle assure ainsi la survie de sa tribu à travers leur descendance, même métissée, et son intégration dans l'identité des nouveaux maîtres. ${ }^{25}$ Son sacrifice légalise, d'une certaine façon, la victoire des nouveaux venus et doit effacer les antagonismes d'hier. ${ }^{26}$ Sont symboliques également les I27 ans de vie que Khaldoun lui prête. ${ }^{27}$ Cela relève avant tout d'une tournure stylistique destinée à souligner l'importance du personnage, à l'exemple des I2O ans prêtés à Moïse dans la Bible. Cela n’empêche pas les romanciers de faire mourir la Kahéna encore dans toute la splendeur de sa beauté !

Les chroniques, avec plus ou moins de détails, présentent cette guerrière comme le dernier rempart contre la conquête de l'Ifriqiya par les Arabes à la fin du viI siècle après la mort, en 688, de Koceïla / Kuseyla, le chef berbère qui avait mis fin à l'avancée triomphale d'Oqba ibn Nafi, en 683, et repris Kairouan, cité où ce dernier avait fondé, sur ce territoire, en 670 , la première mosquée. Pour certains chroniqueurs, la Kahina se serait battue aux côtés de Koceila et ils auraient même été amants. Quand il meurt cinq ans plus tard, elle prend sa relève, après avoir réussi à fédérer des tribus berbères contre les troupes arabes conduites par Hassan. Selon Yves Modéran, l'aventure de la Kahena devrait se placer entre 698 et 702-703. ${ }^{28}$ Après avoir réussi à mettre en échec une première fois l'envahisseur près de l'Oued Nini, elle fait de nombreux prisonniers quelle libère à l'exception d'un seul qu'elle va adopter, Khaled, qui l'aurait trahie en renseignant les Arabes sur ses agissements. À ce sujet, En-Noweiri, parle de la bonté de la Kahina libérant ses prisonniers, avant de dénon-

25 Ibid., p. 139.

26 C'est la thèse défendue par Norman Roth dans "The Kâhina : legendary material in the accounts of the « Jewish berber Queen », The Maghreb Review, Londres, vol. 7, 5-6, 1982, p. 124.

27 Khaldoun, op. cit., tome 3, p. 193.

28 Modéran (Yves), op. cit. 
cer, quelques lignes plus loin, la tyrannie quelle exerce sur les habitants, une fois devenue maîtresse de l'Ifriqiya, notamment lorsqu'elle décide de dévaster le pays pour décourager les envahisseurs. Pour elle, ces derniers ne cherchent qu'à s'emparer des « villes, de l'or et de l'argent ", alors que les Berbères veulent garder les champs pour le pâturage et les cultures. ${ }^{29}$ Finalement vaincue, elle meurt, pour les uns, sur le champ de bataille, glaive à la main ; pour Ibn Khaldoun, décapitée près d'un puits, difficile à situer tant de lieux de l'Aurès portent encore le nom de Bîr-el-Kahina aujourd'hui. ${ }^{30}$ Pour El-Bekri, ${ }^{31}$ c'est à Tabarka, ville côtière du nord-ouest de la Tunisie actuelle, qu'elle est décapitée dans un ravin et que sa tête est ramenée au calife. L'attrait de la légende apportant une plus-value touristique aux lieux supposés historiques, on ne s'étonnera pas qu'un guide de tourisme ${ }^{32}$ rapporte une croyance populaire : blessée, la guerrière se serait fondue dans la nuit et, "parfois le soir, non loin du Marabout de Sidi Amor, une lieue à peine au Sud de Tabarka, près d'un puits où elle se serait donné la mort, l'ombre de la reine erre parmi les bruyères et les typas, sur les rives de l'Oued El Kébir, là tout près de Tabarka. » Mais une telle reine, héritière du long passé de l'Ifriqiya, méritait un lieu grandiose, digne de son destin fabuleux, pour y mourir. L'amphithéâtre romain de Thysdrus (E1 Djem) devient la forteresse où elle s'enferme. Les murs garderaient les traces du dernier combat qu'elle a livré. Ce décor la magnifie et la hisse à la hauteur de la puissance romaine de jadis dont ces pierres furent les témoins.

À ce stade, il n'est plus question de chercher à prouver une quelconque vérité historique. Il suffit de constater que le personnage appartient à un fond culturel que se partagent différentes traditions. Les récits des historiens arabes balancent entre hommages et accusations. La victoire difficile des envahisseurs prouve qu'elle résulte de la volonté d'Allah que rien ne pouvait arrêter. Les qualités de l'adversaire justifient que sa descendance soit intégrée dans les rangs des conquérants. Ses fils iront,

29 En-Noweïri, appendice à l'Histoire des Berbères, traduction de Slane, p. 340-341.

30 Op. cit., tome 1, p. 214.

31 El-Bekri, Description de l'Afrique septentrionale [extrait de Description géographique du monde connu], éd. et trad. en français par le baron de Slane, Alger, 1858-1859 (Journal asiatique, 12-14) ; nouv. éd. Paris, 1913.

32 Guide Hachette Visa, 1994. 
avec les nouveaux maîtres, soumettre l'Espagne. Ainsi se clôt avec cette héroïne un cycle historique.

\section{LA REVIVISCENCE DE LA LÉGENDE}

Si les textes arabes, concernant l'Afrique du Nord étaient connus des érudits européens, l'intérêt porté au passé de celle-ci par les nouveaux maitres de l'Algérie, à partir de I830, puis de la Tunisie à partir de I88I, va générer leur traduction en langue française. Ils permettent à un plus grand nombre de lecteurs de les connaitre et pour certains de les confronter aux traditions orales. William Mac Guckin, baron de Slane (I8oI-1878), Irlandais venu en France pour étudier les langues orientales, réalise la traduction de nombreux textes arabes et s'occupe notamment de l'établissement du catalogue de la bibliothèque d'Alger. Son rôle est important dans la connaissance des textes arabes médiévaux.

Mais les Occidentaux vont élargir les références culturelles pour peindre l'héroïne berbère. Au regard porté par des Musulmans va s'ajouter, parfois pour s'opposer, celui généré par la tradition judéo-chrétienne de la culture européenne. Ainsi, les références bibliques font de la Kahina une " Déborah Berbère ", 33 prophétesse juive qui, selon les chapitres 4 et $5 \mathrm{du}$ Livre des Juges, suscite la résistance de son peuple à l'envahisseur cananéen. Marcelle Magdinier ${ }^{34}$ fait le même rapprochement, doublé de celui avec Judith, qui dans le livre du même nom de l'Ancien Testament, écarte la menace d'une invasion assyrienne en décapitant le général ennemi Holopherne, ce que reprend Jean Hilaire, ${ }^{35}$ assimilant la Kahina au "glaive de Judith ». La reine berbère est ainsi réintégrée dans la tradition judéo-chrétienne à laquelle elle est censée avoir appartenu. Ces références religieuses entrent en résonnance avec les envahisseurs arabes agissant au nom d'Allah.

Parfois l'actualité politique interfère dans les références. Les efforts de l'Église pour se réconcilier avec la République française, avec laquelle elle était en froid à la suite des positions laïques de l'État, aboutissent, en

33 Expression de Georges Marçais (1876-1962), archéologue arabisant, il a consacré plusieurs études importantes à l'art et à l'histoire du Maghreb, notamment à l'Algérie médiévale.

34 Magdinier (Marcelle), 1898-1984, La Kahena, Paris, Calmann-Lévy, 1953.

35 Hilaire (Jean), La Kahena, Rouen, Henri Defontaine Edit. 1918. 
I89o, au « toast d'Alger » ${ }^{36}$ qui, malgré les difficultés persistantes, amorce un rapprochement très relatif, entre Rome et Paris. A terme, on assistera à la canonisation de Jeanne d'Arc (I920). Bientôt, les villes d'Alger, Bône, Philippeville et Oran accueilleront une statue de la nouvelle sainte. C'est dans cette période que paraît un texte anonyme, Une Jeanne d'Arc africaine, épisode de l'invasion des Arabes en Afrique : la Kabina, que Jean Déjeux ${ }^{37}$ date de 1890 , époque où on essaie de donner une assise historique et littéraire aux liens entre la France et l'Algérie. Hélène Stora-Sudaka, en mai I929, dans une conférence sur les " Premières immigrations juives en Berbérie », utilise des références bibliques classiques (rapprochement du don de prophétie de la Kaëna [sic] de celui de Joël dans la Bible, citation empruntée à un poème de Mme Aboulker Benichou qui la qualifie de " Judith ou Débora vivante »). Mais survient une appellation dans l'air du temps, celle de "Jeanne d'Arc berbère ", qui donne une coloration patriotique au propos, confirmée plus loin : «Le croissant s'est levé au lieu du sceptre de la Kaëna superbe. » À son tour, l'étendard de l'islam a pâli « devant une enseigne aux triomphantes couleurs : notre drapeau tricolore », emblème du " génie civilisateur de la France ». Il s'agit bien de célébrer l'œuvre de la colonisation. ${ }^{38}$

La position particulière des écrivains d'Algérie, d'origine juive, s'explique aisément : ils sont passés du statut de dhimmi, avant la conquête, à celui de citoyen français, avec le décret Crémieux de i87o. Ils revendiquent la judaïté de la Kahina et sa latinité, ce qui fait le lien avec la présence française au Maghreb. Ils fixent aussi par l'écrit toute une tradition populaire qui fait de la Kahina une ancêtre glorieuse pour tout un petit peuple marginalisé. C'est sensible dans le recueil La Hara conte, qui rassemble de brefs récits qui circulaient dans ce quartier pauvre où vivaient les Juifs de Tunis. J. Vehel (pour Jacques-Victor Lévy) signe l'un d'eux, « La Belle Kahéna ». Revenant sur la signification de son nom, il prend un plaisir évident à souligner « un point de similitude avec Jeanne d'Arc qui fut, elle aussi, accusée de sorcellerie. » Il renchérit : «Elle était superbe,

36 Mandaté par le Pape Léon Xiır, le cardinal Lavigerie, archevêque d'Alger et de Carthage, participe à l'Amirauté d'Alger à la réception officielle de l'escadre française de la Méditerranée.

37 Déjeux (Jean), Femmes d'Algérie. Légendes, Traditions, Histoire, Littérature, Paris, La Boîte à Documents, 1987, p. 88.

38 Ibid.p. 89-90. 
la Kahéna, dans son armure grise, [...] ayant au côté une petite hache et son épée à garde d'argent ", ajoutant qu'elle " portait à la main une bannière de soie blanche à raies bleues sur laquelle était brodé en lettres d'or le nom de Jéhova ${ }^{39}$. Le récit cumule les superlatifs sur l'héroïne et se conclut sur une phrase enthousiaste : "Quelle légende plus belle que cette incontestable histoire ! $»^{40}$ La proximité presque oxymorique du terme « légende » et de l'adjectif « incontestable » nuance le propos par une note ironique.

L'ascendant du personnage pouvant difficilement se justifier par des pouvoirs magiques à lépoque moderne, les auteurs vont doter l'héroïne d'une beauté extraordinaire. À l'instar de son peuple, elle aura des caractéristiques qui la différencient des Arabes, notamment par sa chevelure " couleur de miel, des yeux couleur de lavande et de métal bleu ${ }^{41}$ Même si Marcelle Magdinier la dote d'une chevelure "d'un noir bleuissant ", " aux grands yeux ténébreux et à la peau bistrée de jolie petite Berbère ». D'une façon générale, elle est éblouissante, comme nimbée de lumière, " telle une vierge de vitrail » écrit Georges Grangean ${ }^{42}$ Les détails s'imposent par leur valeur symbolique, souvent en accord avec la mentalité du moment. Ces détails grâcieux peuvent côtoyer des éléments qui dénotent une forme d'animalité qui se traduit notamment, par une sensualité pour le moins débordante, en particulier chez les auteurs féminins. ${ }^{43}$

Il est un autre moyen de présenter les Berbères, notamment les femmes, comme des êtres irréductibles à la civilisation arabe. Grandjean crée un personnage de femme indomptable, toujours fuyante, et qu'il croie reconnaître sous différentes identités, dont celle de la Kahina. Elle apparât en différents lieux, à des époques éloignées, à la façon d'un mirage. Avec cette personnalité, à la fois pérenne et insaisissable, elle incarne

39 Véhel (J.), « La Belle Kahéna », in La Hara conte... Folklore Judéo-Tunisien, Les Éditions Ivrit, Paris, MCMXXIX, p. 102.

40 Ibid., p. 106.

41 Boisnard (Magali), Le Roman de la Kahéna, Paris, l'Édition de l'art, 1925, p. 42. Gisèle Halimi utilise la même expression pour parler de la couleur des cheveux de la Kahina, mais ses yeux seront verts (La Kabina, Plon, 2006, rééd. 2009, notamment dans la préface du roman).

42 Grandjean (Georges), La Kahena, par l’or, par le fer, par le sang, Aux éditeurs associés, Les éditions du monde modernes, Paris, 1926.

43 Les étreintes sont aussi brûlantes en 1925 (Magali Boisnard) qu’en 2006 (Gisèle Halimi), comme si les auteures s'identifiaient à leur héroïne dont la beauté mature annonce son prochain déclin. 
le combat contre l'envahisseur arabe qui voudrait assigner le féminin à une place obscure dans la société. Façon pour l'auteur de présenter les Français comme des libérateurs. Un certain féminisme, sensible déjà dans les années folles, sous-tend également cette vision qui s'imposera de plus en plus par la suite.

Alors que se déroulent les "événements d'Algérie », qui devaient mener à l'indépendance du pays, La Kahéna, reine des Aurès de Germaine Beauguitte, ${ }^{44}$ défend l'œuvre de la France. Un préfacier reprend l'explication de la conquête arabe au viI ${ }^{\mathrm{e}}$ siècle par l'incapacité des tribus berbères à s'unir politiquement. Un parallèle se fait avec la Régence turque qui n'a pas su assurer la cohésion entre les cités maures et les tribus arabes et berbères, pendant les trois siècles de sa gouvernance. L'absence d'un état-nation aurait conduit la France à intervenir en Afrique du Nord. Implicitement dans ce roman, l'utilisation des villes romaines de Timgad, Djemila, Tipaza, Césarée comme décors de l'action, établit un lien entre l'œuvre romaine et celle entreprise par les Français depuis I830. En I959, quand le roman est publié, les découvertes pétrolières au Sahara font espérer des richesses nouvelles propices au développement du pays. Le souhait est clairement exprimé « que l'œuvre magnifique déjà accomplie, sera poursuivie avec courage et ténacité pour le mieux-être et le bonheur de tous ceux qui peuplent notre belle Algérie française. " Dans le dernier chapitre, "Rencontres dans l'Au-delà », la romancière imagine Saint-Augustin, son « compatriote éminent », accueillant la Khaéna. À son tour, elle recevra Jeanne d'Arc qui annonce : «J'ai bouté l'Anglais hors de France ! », à quoi la Kahéna, répond «Je n'ai pu empêcher l'Arabe de s'implanter dans l'Aurès ». La Pucelle a, en quelque sorte, réparé l'échec de la Berbère, comme la France a mis fin au déclin d'un territoire qui, après la conquête arabe, a été soumis au pouvoir ottoman (I5I2-I830). Une double illustration accompagne le texte : l'une représente "Alger, côte barbaresque qui donnait asile à d'audacieux pirates avant I830 ", l'autre «Le Port d'Alger, un des aspects de la « Nouvelle France », preuve par l'image de l'action civilisatrice de la France.

Dans les mêmes années, la revendication indépendantiste des Algériens s'exprime dans la littérature maghrébine. Le roman de Kateb Yacine,

44 Beauguitte (Germaine), La Kahéna, reine des Aurès, Meaux, Éditions des Auteurs, 1959. 
Nedjma, pose, dès 1956, la question fondamentale de la construction de la nation à travers son histoire, son identité, sa culture. C'est plus tard, en 1974, dans un spectacle intitulé La Guerre de deux mille ans, qu'il utilise le personnage de la Kahina. Le texte sera repris, avec d'autres textes concernant d'autres femmes remarquables par les combats qu'elles ont menés. ${ }^{45}$ Kateb Yacine, étant devenu un opposant au pouvoir tel qu'il s'exerce dans l'Algérie indépendante, prête à la reine berbère les revendications du peuple algérien dont les droits sont bafoués. Pour elle, seule compte «la terre libre d'Amazigh ", les religions ne servant qu'à asservir " aux rois étrangers ». Le seul Dieu est « la terre vivante ». Le pouvoir centralisateur de l'Algérie indépendante, a réveillé les revendications amazighes, notamment sur la reconnaissance du tamazight comme langue officielle, au même titre que l'arabe, revendication qui n'aboutira qu'en 2002. La quatrième de couverture cite l'auteur : « La question des femmes algériennes dans l'histoire [...] m'a toujours parue primordiale », car « c'est la mère qui fait prononcer les premiers mots à l'enfant, c'est elle qui construit son monde ». De ce point de vue, on pourrait affirmer que la Kahina a engendré la nation algérienne, car elle en incarne la combativité inépuisable.

\section{UN MYTHE D'AUJOURD'HUI ?}

Ce lien indéfectible avec la terre, c'est ce que vont découvrir également les Européens installés depuis le XIX ${ }^{\mathrm{e}}$ siècle, dans les pays du Maghreb, comme les populations juives dont la présence était bien antérieure à la colonisation française. À l'indépendance de la Tunisie et de l'Algérie, la douleur de l'exil suscite une littérature qui tente de recréer par les mots l'univers d'autrefois. L'aventure de la Kahina reprend alors sa place dans ce récit mémoriel, car le combat perdu de la reine devient emblématique de leur sort : l'arrachement à la terre natale est vécu comme une défaite.

La singularité de ces œuvres se mesure d'autant mieux si on les compare à La Kabina singulière de Roger Ikor $^{46}$. Cet écrivain n'a jamais

45 Parce que c'est une femme, textes réunis par Zebeïda Chergui, théâtre (entretien de Yacine Kateb avec El Hanar Benali, 1972, La Kabina ou Dibya; Saout Ennissa, La Voix des femmes, Louise Michel et la Nouvelle Calédonie), Paris, Éditions des Femmes - Antoinette Fouque, 2004, 174 p.

46 Ikor (Roger), La Kahina, Paris, Encre, 1979, 212 p. 
vécu au Maghreb et ne découvre l'Algérie qu'en I935, lors d'un voyage et c'est bien plus tard qu'il donne sa version de l'épopée de la reine berbère. Il tourne en dérision tout ce qui a pu être écrit sur elle et népargne ni l'histoire ni les valeurs religieuses, comme s'il voulait tourner une page sur un passé qui n'a plus rien à apporter au présent. Le ton tranche totalement avec d'autres œuvres écrites par ceux qui ont vécu en Algérie ou en Tunisie. Pierre Cardinal, appartenant à une vieille famille pied-noire algéroise, profondément attaché à son pays, même s'il a pris position pour l'indépendance, publie en 1975, un roman intitulé La Kahena ${ }^{47}$. Il n'est nullement question d'une femme mais d'un lieu, une " guelâa ", sorte de grenier fortifié collectif qui, vu de loin, se confond avec la montagne des Aurès. Les rebelles algériens vont en faire un piège pour mettre en déroute les soldats français qui les pourchassent. On serait tenté de voir dans la vieille femme française qui refuse de quitter la terre que ses aïeux ont fait fructifier, comme le lui demande l'armée pour sa sécurité, une réincarnation de la reine berbère. D'autant plus qu'elle est accompagnée de trois enfants qu'elle affirme être les siens, bien qu'ils ne soient apparemment pas européens. Veut-elle croire encore à un monde où la paix ne triompherait pas par les armes mais par l'amour d'une terre commune? La fin tragique reste ambiguë puisque cette femme, comme les Algériens qui ont piégé la grotte et réussi à attirer les soldats français à leur poursuite, meurent dans l'explosion de ce lieu. Seuls survivent les enfants dont on ne sait ce qu'ils deviendront. Une certaine poésie de l'écriture cherche à donner à ce récit une valeur symbolique que le lecteur est libre de choisir : peut-être la nécessaire disparition du passé pour qu'un avenir soit possible?

Une littérature de la diaspora juive tunisienne a trouvé dans la figure de la Kahina une expression forte de l'attachement à ses racines. Georges Memmi dans son roman Qui se souvient du café Rubens? rassemble les souvenirs de son enfance à Tunis, faisant une large place au personnage de sa mère, analphabète mais à la « mémoire infinie. Elle se souvient du roi Salomon [...] elle a bien connu Pharaon d'Égypte, car elle en parle souvent. " «Agée de douze siècles au moins, ma mère se souvient de la 
reine Kahena. ${ }^{48}$ L'amour filial qu'il éprouve maintient le lien avec le pays perdu et avec toute une culture ancestrale dont la reine berbère fait partie au même titre que les références au texte biblique. Les Belles de Tunis de Nine Moati, ${ }^{49}$ raconte la vie de trois générations de femmes en Tunisie, entre 1856 et 1956. Le premier récit concerne le petit peuple de la Hara. Une marieuse vante, au bijoutier des souks qui cherche une épouse, les qualités d'une jeune fille présentée comme une descendante de la Kahe$\mathrm{na}^{50}$ dont la destinée tragique est brièvement rappelée. Lévocation de sa mort s'inscrit clairement dans la filiation du récit de « La Belle Kahena » de J. Vehel qui décrivait la recherche vaine des assaillants de la reine, réfugiée dans la forteresse d'El Djem, jusqu'à ce qu'ils comprennent qu'elle avait dû suivre un souterrain de quelques kilomètres « qui allait jusqu'à la mer, dont les flots berçaient sans doute le corps de la superbes amazone... " ${ }^{51}$ Chez Nine Moati, c'est après un cheminement semblable que les poursuivants découvrent, « un étrange sourire sur les lèvres et ses voiles rouges répandus autour d'elle, la belle Kahena bercée par les flots calmes de la Méditerranée. " ${ }^{52}$ En confrontant ces deux textes, on peut saisir un des mécanismes utilisés dans la transmission orale d'un événement : la reprise d'invariants (le lieu, les circonstances) accompagne des ajouts laissés à la fantaisie du conteur (le corps vu et non pas imaginé, les voiles déployés figurant une sorte de chevelure écarlate qu'on a souvent prêtée au personnage, le sourire perçu comme un défi lancé aux vainqueurs). Autant d'éléments qui relèvent de la mémoire affective : c'est par l'éveil de la sensibilité de l'auditeur, ou du lecteur, qu'un événement, un propos deviennent signifiants et exemplaires. En se répétant, en s'adaptant à différents publics, loin de perdre de leur force, ils gagnent en persuasion et en vérité. Déjà, dans l'exemple précédent, le fils ne peut se détacher des propos de sa mère. La distance tendrement ironique dont il use pour les rapporter souligne combien ils ont bercé son enfance, forgé sa personnalité. Ils continuent à nourrir sa vie.

48 Memmi (Georges), Qui se souvient du café Rubens? Paris, Éditions J.-C. Lattès, 1984, p. 23 et p. 28.

49 Les Belles de Tunis, Éditions du Seuil, 1983.

50 Op. cit., p. 23.

51 J. Véhel, op. cit., p. 106.

52 Les Belles de Tunis, p. 24. 
C'est ce qui manque à La Kabina de Gisèle Halimi. ${ }^{53}$ L'ouvre est hybride, multipliant les références historiques (avérées par huit pages de bibliographie, ce qui est inusité pour un roman), mêlées à une aventure totalement romanesque, au sens d'imaginaire, puisque nous ne perdons aucun élément des pensées intimes de l'héroïne dont pourtant rien n'est attesté. Surtout, on s'aperçoit vite que celle qui s'exprime, c'est l'avocate elle-même. D'ailleurs, elle fait de ce roman le troisième volet d'une trilogie commencée avec La Cause des Femmes (1973), puis Le lait de l'oranger (1988). Autrement dit, ce roman coiffe les récits de ses combats féministes, de sa jeunesse tunisienne, pour les couronner par l'image magnifiée de sa propre personne. Son grand-père ne lui disait-il pas qu'elle avait les mêmes cheveux couleur de miel de la Kahina ? Cela frôle parfois le ridicule quand, usant des dons de prophétesse de l'héroïne, elle lui fait prédire qu'un jour, Arabes et Juifs vivront sur la même terre que l'on devine être celle de la Palestine. Ce n'est pas le contenu du propos qui surprend, mais le procédé littérairement contre-productif, car ce n'est plus la reine qu'on entend, mais la femme engagée dans les combats politiques du $\mathrm{Xx}^{\mathrm{e}}$ siècle réaffirmant ses positions militantes.

\section{UN MYTHE MÉMORIEL}

Pour Norredine Sabri, la Khéna ne peut accéder au statut de mythe, car elle « demeure prisonnière du particulier et du régional, parce que trop ancrée dans l'histoire maghrébine. ${ }^{54}$. Il est vrai que ce contexte est omniprésent, mais nuancé par les circonstances. Cette reine a été " adoptée » par au moins trois communautés différentes. Elle inspire toujours les romanciers, preuve de son éternelle actualité : La Kabéna de la Courtille $e^{55}$ évoque le combat d'une jeune immigrée vivant en France, dont les parents sont assassinés lors d'un voyage en Algérie, pendant la " décennie noire ». Aussitôt, elle décide de les venger en poursuivant tous les adeptes du FIs. La Kahéna désigne aussi le nom d'une maison qui a appartenu à un maire français de Cyrtha, au temps de la colonisation, mais qui renferme des éléments qui permettent au héros (et au lecteur)

53 Halimi (Gisèle), La Kabina, Paris, Plon, 2006 ; rééd. Pocket, 2009.

54 Sabri (Norredine), op. cit., p. 261.

55 Berkani (Berri), La Kahéna de la Courtille, « Ecritures berbères », Paris, L'Harmattan, 2002. 
de reconstituer tout le passé douloureux de l'Algérie : pour l'auteur, seule la mémoire peut construire, par-delà les saccages, un monde durable. ${ }^{56}$ Il y a donc une " plasticité » dans cette référence à l'hérö̈ne berbère qui la dote d'un universalisme indéniable : la perte de l'identité, le combat pour préserver sa liberté, le besoin de préserver ses racines se retrouvent dans l'expérience de toutes les nations.

La Kahina ne serait-elle pas le mythe d'une mémoire blessée?

BIBLIOGRAPHIE SUR LA KAHINA

\section{A / Sources historiques}

El-Bekri, Description de l'Afrique septentrionale [extrait de Description géographique du monde connu], éd. et trad. en français par le baron de Slane, Alger, 1858-1859 (Journal asiatique, 12-14) ; nouv. éd. Paris, 1913.

Iвn Khaldoun, Histoire des Berbères et des dynasties musulmanes de l'Afrique septentrionale, traduite par le baron de Slane, Alger, imprimerie du Gouvernement, 1852.

Julien (Charles-André), Histoire de l'Afrique du Nord. Tunisie - Algérie - Maroc de la conquête arabe à 1830, t. 2, Paris, Payot, rééd. 1975.

Lugan (Bernard), Histoire des Berbères. Un combat identitaire plurimillénaire, Bernard Lugan Éditeur, 2012.

Masqueray (Émile), Formation des cités chez les populations sédentaires de l'Algérie (Kabyles du Djurdjura, Chainïa de l'Aourâs, Beni Mezâb), Paris, E. Leroux, 1886 ; Éd. Fanny Colonna, Aix-en-Provence, Edisud, 1983.

Meynier (Gilbert), L'Algérie des origines. De la prébistoire à l'avènement de l'islam, La Découverte/Poche, 2010.

\section{B / Études sur la Kahina}

Camps (Gabriel), L'Afrique du nord au féminin, Librairie Académique Perrin, 1992. Dejeux (Jean), Femmes d'Algérie. Légendes, Traditions, Histoire, Littérature, Paris, La Boîte à Documents, 1987.

Modéran (Yves), « Kahena ", in Encyclopédie berbère, 27 | Kairouan - Kifan Bel-Ghomari [En ligne], mis en ligne le 01 juin 2011, consulté le 25 avril 2018. URL : http://journals.openedition.org/encyclopedieberbere/1306

56 Bachi, (Salim), La Kahéna, Paris, Gallimard, 2003. 
Slousch (Norbert), «La race d'El Cahena », in La Revue indigène, n 44, déc. 1909 , p. 580-581.

Roth (Norman), "The Kâhina : legendary material in the accounts of the « Jewish berber Queen », The Maghreb Review, Londres, vol. 7, 5-6, 1982.

SABri (Norredine), La Kahéna. Un mythe à l'image du Maghreb, Paris, L'Harmattan, «Critiques littéraires », 2011.

TALBI (Mohamed), «Un nouveau fragment de l'histoire de l'Occident musulman l'épopée d'A1-Kahina », in Les Cabiers de Tunisie, t. xıx, $1^{\text {er }}$ et $2^{\text {e }}$ trim., 1971.

\section{C / Euvres littéraires}

BACHi, (Salim), La Kahéna, Paris, Gallimard, 2003.

Berkani (Berri), La Kahéna de la Courtille, «Écritures berbères », Paris, L'Harmattan, 2002.

Beauguitte (Germaine), La Kahéna, reine des Aurès, Meaux, Éditions des Auteurs, 1959.

Boisnard (Magali), Le Roman de la Kahéna, Paris, l'Édition de l'art, 1925.

Cardinal (Pierre), La Kahena, Paris, Julliard, 1975.

Grandjean (Georges), La Kahena, par l'or, par le fer, par le sang, Aux éditeurs associés, Les éditions du monde modernes, Paris, 1926.

Halimi (Gisèle), La Kabina, Plon, 2006, rééd. 2009.

Hilaire (Jean), La Kabena, Rouen, Henri Defontaine Édit. 1918.

Ikor (Roger), La Kabina, Paris, Encre, 1979.

Magdinier (Marcelle), 1898-1984, La Kahena, Paris, Calmann-Lévy, 1953.

Mеммі (Georges), Qui se souvient du café Rubens ? Paris, Éditions J.-C. Lattès, 1984.

Moati (Nine), Les Belles de Tunis, Éditions du Seuil, 1983.

Parce que c'est une femme, textes réunis par Zebeïda Chergui, théâtre (entretien de Yacine Kateb avec El Hanar Benali, 1972, La Kabina ou Dibya ; Saout Ennissa, La Voix des femmes, Louise Michel et la Nouvelle Calédonie), Paris, Éditions des Femmes - Antoinette Fouque, 2004.

VéHel (Jacques), « La Belle Kahéna », in La Hara conte... Folklore Judéo-Tunisien, Les Éditions Ivrit, Paris, McMxxix. 